\title{
Cause of the fragile-to-strong transition observed in water confined in C-S-H gel
}

\author{
Manuel Monasterio, ${ }^{1}$ Helén Jansson, ${ }^{2}$ Juan J. Gaitero, ${ }^{3}$ Jorge S. Dolado, ${ }^{3}$ \\ and Silvina Cerveny ${ }^{1,4, a)}$ \\ ${ }^{1}$ Centro de Fisica de Materiales (CSIC, UPV/EHU)-Materials Physics Center (MPC), Paseo Manuel \\ de Lardizabal 5, 20018 San Sebastián, Spain \\ ${ }^{2}$ Department of Civil and Environmental Engineering, Chalmers University of Technology, Göteborg, Sweden \\ ${ }^{3}$ Tecnalia Research \& Innovation, Parque Tecnológico de Vizcaya, Edif. 700, 48160 Derio, Spain \\ ${ }^{4}$ Donostia International Physics Center, 20018 San Sebastián, Spain
}

(Received 7 June 2013; accepted 10 October 2013; published online 31 October 2013)

\begin{abstract}
In this study, the rotational dynamics of hydration water confined in calcium-silicate-hydrate (C-S-H) gel with a water content of $22 \mathrm{wt} . \%$ was studied by broadband dielectric spectroscopy in broad temperature $(110-300 \mathrm{~K})$ and frequency $\left(10^{-1}-10^{8} \mathrm{~Hz}\right)$ ranges. The C-S-H gel was used as a $3 \mathrm{D}$ confining system for investigating the possible existence of a fragile-to-strong transition for water around $220 \mathrm{~K}$. Such transition was observed at $220 \mathrm{~K}$ in a previous study [Y. Zhang, M. Lagi, F. Ridi, E. Fratini, P. Baglioni, E. Mamontov and S. H. Chen, J. Phys.: Condens. Matter 20, 502101 (2008)] on a similar system, and it was there associated with a hidden critical point of bulk water. However, based on the experimental results presented here, there is no sign of a fragile-to-strong transition for water confined in C-S-H gel. Instead, the fragile-to-strong transition can be explained by a merging of two different relaxation processes at about 220 K. () 2013 AIP Publishing LLC. [http://dx.doi.org/10.1063/1.4826638]
\end{abstract}

\section{INTRODUCTION}

Studies on the structural and dynamical properties of supercooled water (at temperatures lower than its freezing point, $273 \mathrm{~K}$ ) have in recent years most often been motivated by its probable role in biological activities of cells, proteins or DNA. However, low temperature properties of water are difficult to study due to the homogeneous nucleation temperature at $\mathrm{T} \cong 235 \mathrm{~K}(1 \mathrm{~atm})$, which defines a limit below which bulk liquid water cannot be experimentally studied.

Among a number of atypical properties of bulk water, ${ }^{1}$ a phase transition from a high density to a low density liquid (the liquid-liquid critical point hypothesis ${ }^{2}$ in the supercooled liquid region of the water phase diagram) in the temperature range of $220-230 \mathrm{~K}$ was predicted. ${ }^{3}$ This phase transition is supposed to emerge as a sudden change in the water dynamics, at some point, in the temperature range where water normally crystallizes. Nevertheless, crystallization of water can be avoided by surface interactions or by confining it in small cavities (with a characteristic size of tens of $\AA$ ) and, therefore, some authors have tried to verify this hypothesis by study confined water, instead of bulk water, and from the results the existence of a "fragile-to-strong" transition of confined water at about $220 \mathrm{~K}$ have been proposed. However, this fragile-to-strong scenario has been questioned and, despite numerous discussions, still an agreement has not been reached. It should, however, be noted that confinements always introduce surfaces, which, in turn, have an influence on, as well as modify, the water dynamics. Therefore, all ex-

a)E-mail: scerveny@ehu.es trapolations of such observations to the properties of the bulk state should be done very carefully.

The fragile-to-strong scenario and its possible origin have been widely debated in the literature. From quasi-elastic neutron scattering (QENS) studies it has been observed that the relaxation time of confined water exhibit an abrupt crossover from a non-Arrhenius to an Arrhenius dependence as the temperature decreases, which has been associated with the appearance of the fragile-to-strong transition (FST) expected for bulk water. The sharp change in temperature behavior has been observed for water in a variety of confining systems such as MCM- $41,{ }^{4}$ carbon nanotubes, ${ }^{5}$ white cement ${ }^{6}$ as well as in aqueous solution of DNA, ${ }^{7}$ RNA, ${ }^{8}$ and lysozyme. ${ }^{9}$ Some of these, or similar confining systems, have also been studied by using other different experimental techniques, such as broadband dielectric spectroscopy (BDS), ${ }^{10-15} \mathrm{nu}$ clear magnetic resonance (NMR), ${ }^{16}$ or a combination of different techniques. ${ }^{17-22}$ In such studies, most likely due to the broadband frequency range analyzed such crossover for water was either not observed, or attributed to other causes different to that of the fragile-to-strong transition scenario. Thus, no correlation was found by studies of the same sample by QENS and other techniques.

As mentioned above, one of the systems investigated by QENS was hydrated cement in which an abrupt crossover was shown to occur for confined water. ${ }^{6}$ Cement is a multicomponent material (mainly consisting of calcium silicates $\left(\mathrm{C}_{2} \mathrm{~S}\right.$ and $\left.\mathrm{C}_{3} \mathrm{~S}\right)$, aluminates and alumino ferrites). When the cement grains are hydrated mostly exothermic reactions occur and a three-dimensional network is developed in which some of the water becomes a part of the hydration products whereas other water molecules, which are in excess, are 
confined in pores of different sizes. The mechanism of cement hydration is quite complex and it is still not fully understood $^{23,24}$ in spite of the efforts made from both experiments and simulations. ${ }^{25,26}$ The major hydration product is calcium silicate hydrate (C-S-H gel), which also is the main source for the strength of concrete. C-S-H gel is a poorly crystalline nanostructured material formed by silicate chains stabilized by calcium ions and hydroxyl groups. The excess water, which is an integral part of the C-S-H network, is generally located in small nanometric pores $(1-3 \mathrm{~nm})$, larger pores $(3-10 \mathrm{~nm})$, or in capillary pores (even larger than $10 \mathrm{~nm}$ ). A detailed description of the different models of the structure of the calcium silicate hydrate gel can be found in Refs. 23-34.

In a previous work, we investigated the rotational properties of water molecules confined in C-S-H gel ${ }^{35}$ of different water contents (from $6 \mathrm{wt} . \%$ to $15 \mathrm{wt} . \%$, where water remains amorphous even at low temperatures) by BDS, $\left(10^{-2}-10^{6} \mathrm{~Hz}\right)$ in the temperature range of $110-230 \mathrm{~K}$. We found three relaxation processes (labeled 1-3 from the fastest to the slowest) and it was shown that the temperature dependence of the relaxation time of process 2 presented a crossover at $\mathrm{T}=145 \mathrm{~K}$. However, it should be noted that this crossover is not related to the apparent fragile-to-strong transition detected by QENS (mentioned above), since it appears at both lower temperatures and on a slower time scale than that transition. ${ }^{35}$ The origin of this crossover was instead shown to be due to confinement effects. ${ }^{35}$ However, in our previous work we did not perform experiments in the high temperature region where the crossover associated with the fragile-to-strong transition is generally observed for water confined in white cement as well as in other types of confinements.

In this work, we have therefore analyzed the dynamics of water confined in C-S-H gel in both a broader frequency $\left(10^{-1}-10^{8} \mathrm{~Hz}\right)$ and temperature $(110-300 \mathrm{~K})$ range using broadband dielectric spectroscopy and differential scanning calorimeter (DSC). In addition, we explore a higher water content than in our previous work $\left(c_{w}=22 \mathrm{wt} . \%\right.$, expressed as grams of water/grams of dry cement) since it enhances the sensitivity at higher frequencies. The results are presented in two different ways:

(1) Analysis of the dielectric permittivity in the high frequency range $10^{6}-10^{8} \mathrm{~Hz}$.

(2) Including also lower frequencies and analyze the data in the broad frequency interval $10^{-1}-10^{8} \mathrm{~Hz}$.

This twofold analysis method reveals that: (1) only considering a restricted frequency interval, the relaxation time seems to exhibit a clear crossover at $\mathrm{T}=220 \mathrm{~K}$, and thereby, we are able to reproduce the exact relaxation map obtained in Ref. 6. (2) Including also lower frequencies, i.e., investigating a broader frequency range, the suggested fragile-to-strong transition for water confined in C-S-H gel cannot be observed. Instead, two different well-resolved processes (denoted 1 and 2) are obtained. Obviously, the limitation of the frequency window is the origin of the apparent fragile-to-strong transition, i.e., the crossover in temperature dependence at $210 \mathrm{~K}$ obtained by QENS. In contrast to the origin of pro- cess 2, which is known to be due to the dynamics of water molecules in small pores, the origin of process 1 is not established. In this work, we will show that the fast process 1 in cementitious materials might be related to a relaxation process that is observed in other systems in which hydroxyl groups are present and of relative importance.

\section{EXPERIMENTAL}

The C-S-H gel investigated was prepared by mixing $5 \mathrm{~g}$ of pure tricalcium silicate $\left(\mathrm{C}_{3} \mathrm{~S}\right)$ with $1400 \mathrm{~g}$ of distilled water. The great water to $\mathrm{C}_{3} \mathrm{~S}$ ratio served to maintain the calcium concentration in the solution low enough to prevent portlandite precipitation, but without affecting to the formation of $\mathrm{C}-\mathrm{S}-\mathrm{H}$ gel. The resulting dispersion was sealed in a container to avoid carbonation, and after 39 days of continuous stirring at room temperature, the dispersion was filtered and the obtained solid material was dried in an oven at $60^{\circ} \mathrm{C}$ for an hour. The resulting C-S-H gel had a water content of $c_{w}=22 \mathrm{wt} . \%$.

A broadband dielectric spectrometer, Novocontrol Alpha-N, was used to measure the complex dielectric permittivity $\varepsilon^{*}(\omega)=\varepsilon^{\prime}(\omega)-i \varepsilon^{\prime \prime}(\omega), \omega=2 \pi f$, in the frequency range $10^{-1}-10^{6} \mathrm{~Hz}$. The isothermal frequency scans were performed at every 5 th degree in the temperature range $120-230 \mathrm{~K}$. For higher frequencies $\left(10^{6}-10^{8} \mathrm{~Hz}\right)$ an Agilent $\mathrm{RF}$ impedance analyzer 4192B was used. In this case the isothermal frequency scans were performed at every 2.5 th degree in the temperature interval 190-230 K. For each measurement, the sample temperature was controlled by a nitrogen gas flow with a stability better than $\pm 0.1 \mathrm{~K}$. For both types of measurements parallel gold plated electrodes (with a diameter of $30 \mathrm{~mm}$ and of $10 \mathrm{~mm}$ for the low and high frequency range, respectively) were used. The sample thickness was typically of $0.1 \mathrm{~mm}$ for both low and high frequency measurements.

Standard calorimetric measurements (DSC) were performed by means of a Q2000TA Instrument, using cooling and heating rates of $10 \mathrm{~K} / \mathrm{min}$. Hermetic aluminum pans were used for all measurements and the weight of each sample was about $10 \mathrm{mg}$. Thermo-gravimetric analyses were done by using a TGA-Q500 (TA Instruments). All the measurements were conducted under high purity nitrogen flow over a temperature range of $30-800^{\circ} \mathrm{C}$ with a ramp rate of $5 \mathrm{~K} / \mathrm{min}$.

\section{RESULTS}

Figure 1 shows the calorimetric response of the sample CSH-22 $\left(c_{w}=22\right.$ wt. $\left.\%\right)$. On cooling, a main peak with a maximum at about $230 \mathrm{~K}$ is observed representing ice formation. In general, in large size confinements and/or at high water contents crystallization is not always totally prevented but the freezing point is shifted to lower temperatures. The depression of the freezing point with approximately $40 \mathrm{~K}$, which is in agreement with previous observations for water in white cement, ${ }^{6}$ gives an indication on that the freezable water is located in pores larger than $3 \mathrm{~nm} .{ }^{36}$ This result is similar to previous observations for white cement. ${ }^{6}$ As obtained by integration, the area of this exothermic peak corresponds to $\sim 3 \mathrm{wt} . \%$ of the total water content. 


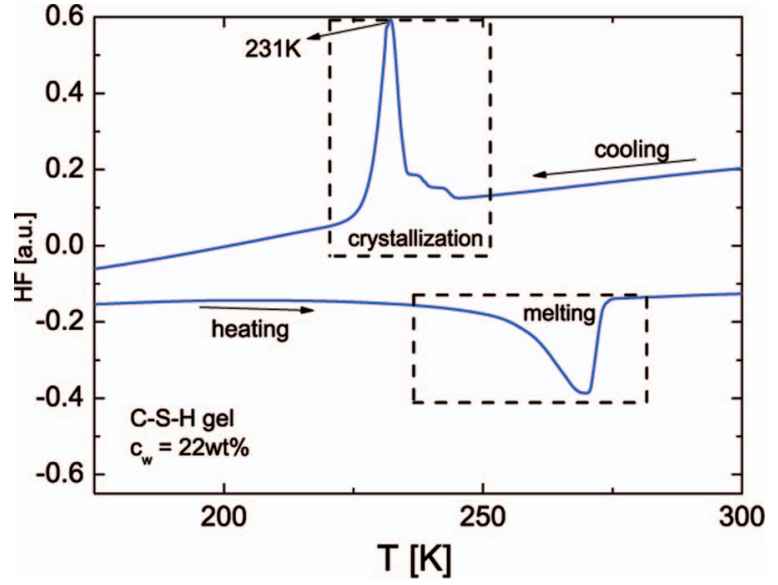

FIG. 1. DSC heat flow curve obtained for the sample CSH-22. The exothermic and endothermic features displayed at cooling and heating is due to crystallization and melting of water, respectively.

\section{A. Dielectric relaxation spectra in the frequency range $10^{6}-10^{8} \mathrm{~Hz}$}

Figure 2(a) shows the imaginary part $\left(\varepsilon^{\prime \prime}\right)$ of the complex permittivity at different temperatures measured (190-225 K) in the frequency range $10^{6}-10^{8} \mathrm{~Hz}$ obtained for the $\mathrm{CSH}-22$ sample. In this frequency region one single peak is observed at all temperatures, which was fitted with a symmetrical ColeCole (CC) function ${ }^{37}$

$$
\varepsilon^{*}(\omega)=\varepsilon_{\infty}+\frac{\Delta \varepsilon}{\left[1+(i \omega \tau)^{\alpha}\right]},
$$

where $\alpha$ is a shape parameter $(0<\alpha<1), \Delta \varepsilon=\varepsilon_{s}-\varepsilon_{\infty}$ is the relaxation strength; $\tau$ is a relaxation time; and $\omega$ is the angular frequency. Additionally, a power law term was added to account for the apparent conductivity contribution at low frequencies. In Figure 2(a) the result of the fitting is shown by solid lines.

The temperature dependence of the obtained relaxation time $(\tau)$ is shown in Figure 2(b) by red squares. The time scale of this relaxation exhibits an Arrhenius-type temperature dependence (Eq. (2)) below approximately $T=(210$ $\pm 5) \mathrm{K}$. At higher temperatures a clear deviation from the Arrhenius behavior is observed. This behavior is in good agreement with that obtained for white cement by quasielastic neutron scattering (QENS) ${ }^{6}$ (see blue triangles in Figure 2(b)). It is important to remark that the QENS relaxation times were obtained at four different $Q$ values $\left(0.2,0.4,0.6\right.$, and $\left.0.8 \AA^{-1}\right)$ simultaneously analyzed to extract the averaged relaxation time, $\tau$. In addition, it is indicated that the relaxation times are almost Q-independent. ${ }^{6}$ Moreover, a comparison between dielectric and QENS relaxation times can be made since it is well-established that the time scale deduced from neutron scattering at a momentum transfer $Q$ of about $0.9 \AA^{-1}$ is comparable with that obtained by dielectric spectroscopy. ${ }^{38,39}$ As seen in Figure 2(b), the time scale obtained from QENS is in perfect agreement to the corresponding time scale of the dielectric relaxation, as expected for the $Q$ values analyzed.
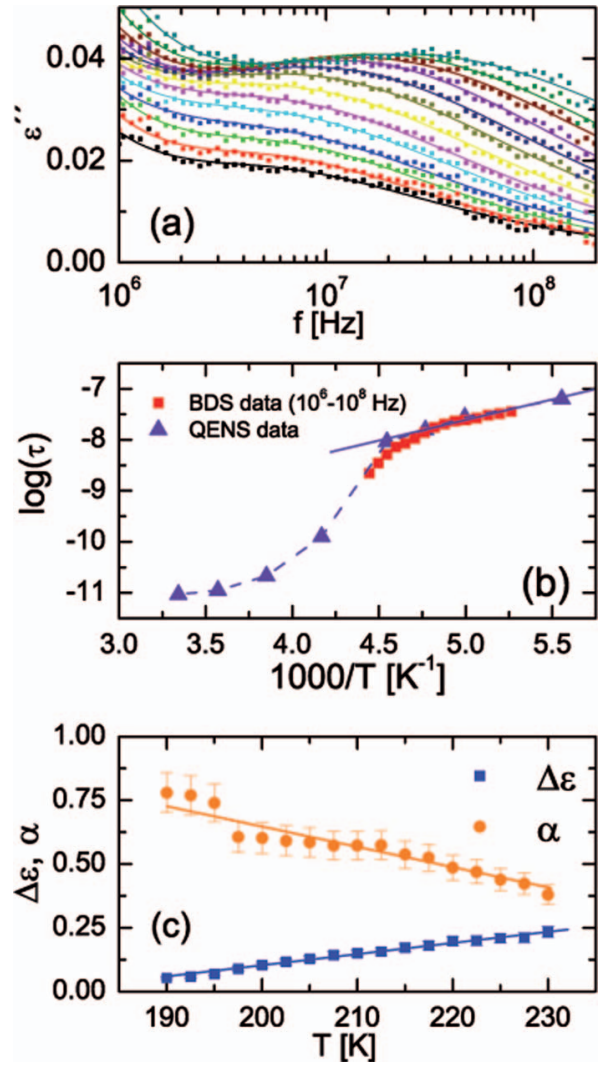

FIG. 2. (a) The loss component, $\varepsilon^{\prime \prime}$, of the complex dielectric permittivity $\left(\varepsilon^{*}(\mathrm{f})\right)$ for CSH-22 at different temperatures. An apparent single dielectric process is observed in this frequency range. The solid line shows the leastsquares fit of a superposition of a power law for conductivity and a Cole-Cole function for the imaginary part. (b) Temperature dependence of the relaxation time $\tau(T)$ obtained from this fitting (red squares) compared to the relaxation time of white cement (blue triangles) obtained from Ref. 6. The blue solid and dotted lines represent the Arrhenius and the VFT fitting of that sample, respectively. (c) Dielectric strength $(\Delta \varepsilon)$ and shape factor $(\alpha)$ as a function of the temperature. Errors bars are added on this plot.

Below $210 \mathrm{~K}$, the relaxation times are fitted by the Arrhenius equation,

$$
\tau(T)=\tau_{o} \exp \left(\frac{E_{a}}{k T}\right),
$$

where $E_{a}$ represents a mean activation energy, $k$ is the Boltzmann's constant, and $\tau_{o}$ is related to a molecular vibration time. Values of $E_{a}$ and $\log \tau_{o}$ are $0.16 \mathrm{eV}$ and $-11.70 \mathrm{~s}$, respectively. Finally, temperature dependences of the relaxation strength $(\Delta \varepsilon)$ and shape parameter $(\alpha)$ are shown in Figure 2(c). Both parameters follow a smooth behavior, $\Delta \varepsilon$ and $\alpha$ increasing and decreasing with temperature, respectively, i.e., neither the shape parameter nor the relaxation strength changes its behavior at the crossover in temperature dependence around $\mathrm{T}=210 \mathrm{~K}$.

\section{B. Dielectric relaxation spectra in a broad frequency range $10^{-1}-10^{8} \mathrm{~Hz}$}

The situation becomes different when analysing the same sample in the broader frequency range $10^{-1}-10^{8} \mathrm{~Hz}$, i.e., including also lower frequencies. Figures 3(a) and 3(b) show 

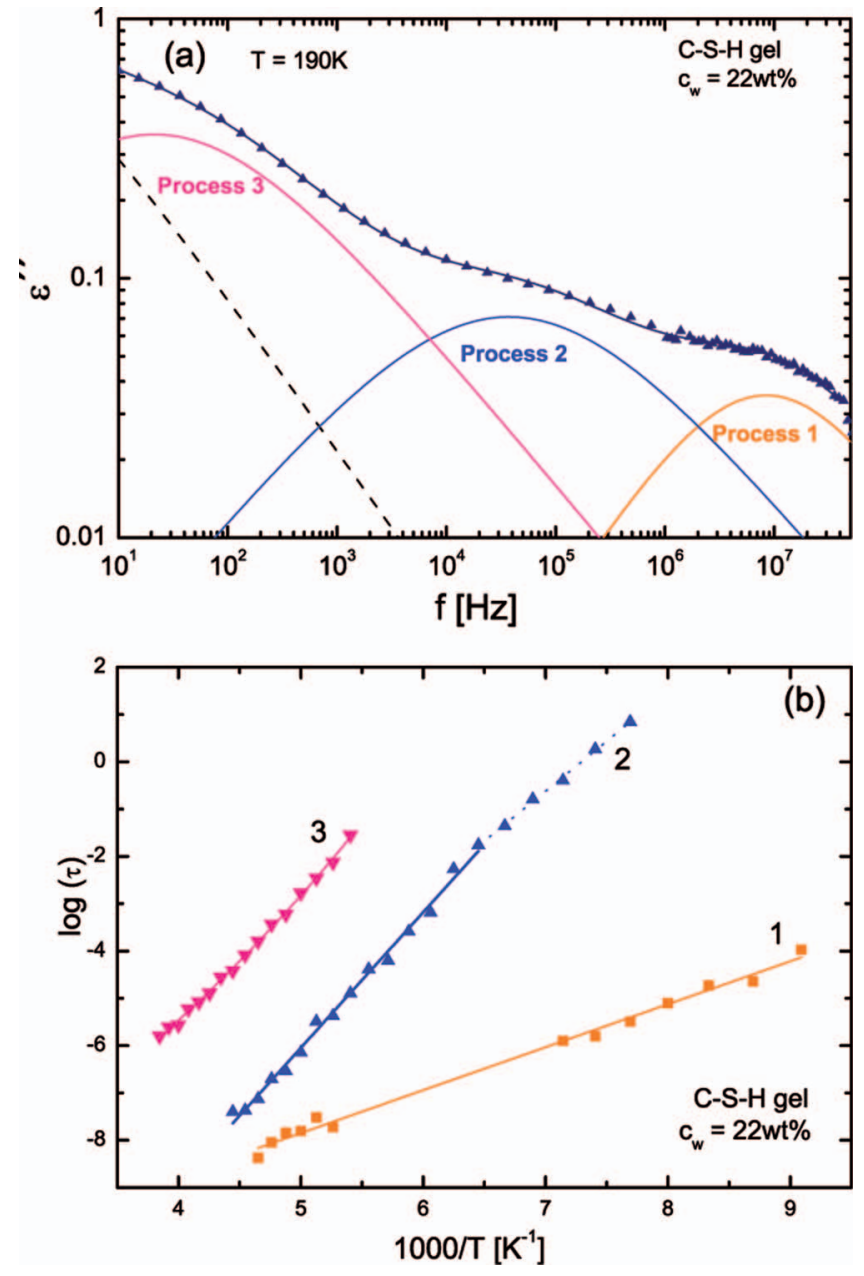

FIG. 3. (a) Loss component, $\varepsilon^{\prime \prime}$, of the complex dielectric permittivity $\left(\varepsilon^{*}(f)\right)$ obtained for CSH-22 at $190 \mathrm{~K}$. The solid line through the data points shows the superposition of three Cole-Cole functions each describing the different processes 1,2 , and 3 (as described in the text) and the conductivity contribution (dashed line). (b) The temperature dependence of the relaxation time $\tau(T)$ obtained from the fitting.

the imaginary part $\left(\varepsilon^{\prime \prime}\right)$ of the complex permittivity at $190 \mathrm{~K}$. In this case, we observe three dielectric processes (called process 1,2 , and 3 from the fastest to the slowest relaxation). Since all the obtained dielectric processes in the C-S-H gel are symmetric, we have used a superposition of three ColeCole (CC) functions (Eq. (1)) plus a conductivity contribution (at low frequencies and high temperatures) to fit the dielectric spectrum at each temperature. More details of the fitting procedure can be found in Ref. 35. Figure 3(a) shows the result of the total fitting as well as the contribution of the three different processes separately at $\mathrm{T}=190 \mathrm{~K}$.

The temperature dependence of the relaxation time is shown in Figure 3(b). The relaxation time of process 1 follows an Arrhenius behavior in the entire temperature range, with an activation energy of $0.18 \mathrm{eV}$ and $\log \left(\tau_{0}\right)=-12.4$. Process 2 presents a crossover from high temperature nonArrhenius behavior to low temperature Arrhenius behavior at a temperature of $T_{\text {cross }}=155 \mathrm{~K}$. Table I shows activation energies and the pre-exponential factor for all water contents for process 2 . Process 3 shows a non-Arrhenius behavior which
TABLE I. Activation energy $E_{\mathrm{a}}$ and pre-exponential factor $\left(\log \left(\tau_{o}(\mathrm{~s})\right)\right.$ corresponding to process 2 for data in Figure A of the supplementary material. ${ }^{42}$

\begin{tabular}{lccc}
\hline \hline \multirow{2}{*}{ Sample } & $c_{w}(\mathrm{wt} . \%)$ & \multicolumn{2}{c}{ Process 2} \\
\cline { 3 - 4 } & & $\log \left(\tau_{o}(\mathrm{~s})\right)$ & $E_{a}(\mathrm{eV})$ \\
\hline CSH-06 & 6.25 & $-16.9 \pm 0.1$ & $0.37 \pm 0.02$ \\
CSH-09 & 9.75 & $-17.3 \pm 0.1$ & $0.39 \pm 0.02$ \\
CSH-11 & 11.00 & $-16.5 \pm 0.4$ & $0.39 \pm 0.02$ \\
CSH-15 & 15.10 & $-17.0 \pm 0.2$ & $0.43 \pm 0.02$ \\
CSH-22 & 22.00 & $-15.4 \pm 0.2$ & $0.41 \pm 0.03$ \\
\hline \hline
\end{tabular}

can be fitted with a single VFT equation, ${ }^{40}$

$$
\tau_{\alpha}=\tau_{o} \exp \left(D T_{o} / T-T_{o}\right),
$$

where $\tau_{o}$ is the relaxation time in the high temperature limit, $D$ is a parameter related to fragility and $T_{0}$ is the temperature at which the dynamics of the system diverge) in the entire temperature range, giving $\tau_{\mathrm{o}}=-12.4 \mathrm{~s}, \mathrm{~T}_{\mathrm{o}}=69 \mathrm{~K}$, and $\mathrm{D}=41.5$. All these results are consistent with the previous results at lower hydration level. ${ }^{35}$ Finally, Figure 4 shows the comparison of the relaxation times obtained from both the restricted frequency and the broad frequency interval, as well as the relaxation time obtained by QENS in Ref. 6. In Sec. IV, we will discuss all the characteristics of these processes.

\section{DISCUSSION}

\section{A. Assignment of dielectric relaxation processes in C-S-H gel}

Although the actual microstructure of C-S-H has not yet been fully resolved, it is known to contain capillary pores (pore diameter larger than $10 \mathrm{~nm}$ ) and gel pores (pore diameter smaller than $10 \mathrm{~nm}) .^{41} \mathrm{It}$ is also worth to mention that the most recent colloidal model (CM), proposed by Jennigs, ${ }^{30,31}$ describes the C-S-H gel as a colloidal particle (globules)

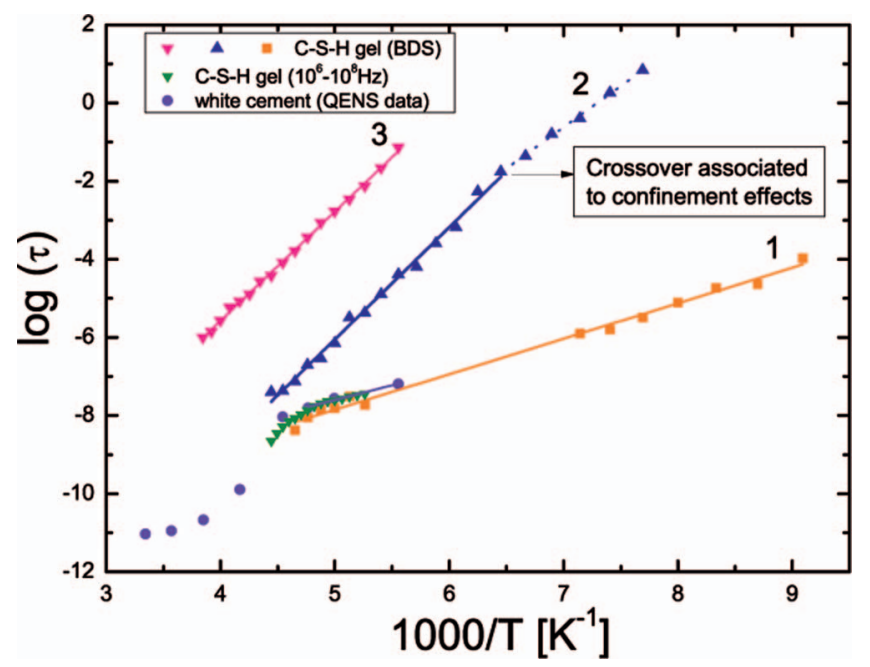

FIG. 4. Comparison of the relaxation times of $\mathrm{CSH}-22$ and that obtained by QENS $^{6}$ with that obtained for the two different analysis approaches shown in figures 2 and 3 of this work. 
having a size of $4 \mathrm{~nm}$ with a layered internal structure, which is in agreement with other models in the literature (see Refs. 27, 28, 32, and 33)]. During the hydration process, these globules form clusters in which a porous structure with two different characteristic lengths is produced: small gel pores (dimensions 1-3 nm) and large gel pores (dimensions 3-12 $\mathrm{nm}$ ). However, regardless of the model considered, the structure of C-S-H gel is highly complex and comprises pores of different sizes in which water molecules can be located.

The strategy of our previous work ${ }^{35}$ was to analyze a fixed structure (developed for C-S-H after 39 days of curing) and progressively dehydrated samples. This procedure allows the identification of the different population of water in the samples. Thus, we have studied the dielectric response of C$\mathrm{S}-\mathrm{H}$ gel at different hydration levels (from $6 \mathrm{wt} . \%$ to $15 \mathrm{wt} . \%$ ) in the frequency window $10^{-1}-10^{6} \mathrm{~Hz} .{ }^{35} \mathrm{We}$ found three dielectric processes $(1,2$, and 3 from the fastest to the slowest), which are all related with the water contained in capillary and gels pore. The molecular origin of process 2 was concluded to be due to water molecules confined in small pores of 1-2 nm size of the microstructure of the C-S-H gel (39 days of curing). For this process, at higher water contents ( $w_{c} \geq 15$ wt.\%, i.e., at water contents higher than the formation of one water monolayer on the pore walls), a crossover in temperature dependence from a non-Arrhenius to an Arrhenius of the relaxation time at $155 \mathrm{~K}$ was observed. This crossover was related with the appearance of finite size effects. ${ }^{35}$ Process 3 was attributed to water molecules located in larger pores of the $\mathrm{C}-\mathrm{S}-\mathrm{H}$ gel $(>3 \mathrm{~nm})$. The assignment of process 1 was, however, not obvious but it was shown that this process was influenced by the number of water molecules since its intensity was slightly increasing with increasing water concentration. In our previous study, it was suggested to be related with the rotation of some intrinsic hydrated dipolar group in the C-S-H gel.

In this work we have studied a sample with an even higher water content $\left(c_{w}=22\right.$ wt. $\left.\%\right)$ with the aim to probe the dynamics in a broader frequency range. With the choice of higher water content, the intensity of both processes 1 and 2 are high enough to be explored also at higher frequencies. When consider the measurements of $c_{w}=22 \mathrm{wt} . \%$ in the frequency interval $10^{-1}-10^{8} \mathrm{~Hz}$, the three observed dielectric processes agree well with those studied at lower concentrations. ${ }^{35}$ The temperature dependence of the relaxation times at different water concentrations is shown in Figure $\mathrm{A}$ of the supplementary material ${ }^{42}$ where it is possible to observe that process 2 and process 3 become slower and faster, respectively, with increasing water content, indicating that each of these processes are due to water molecules in different environments.

\section{B. Crossover at $\mathrm{T}=145 \mathrm{~K}$ associated to finite size effects}

The change in temperature behavior of the relaxation time of process 2 at about $145 \mathrm{~K}$, resembles the VFT to Arrhenius crossover, expected for the $\alpha$-relaxation of glass forming liquids, if the confinement is severe enough. ${ }^{43,44}$ This effect can be rationalized in the framework of the cooperativ-

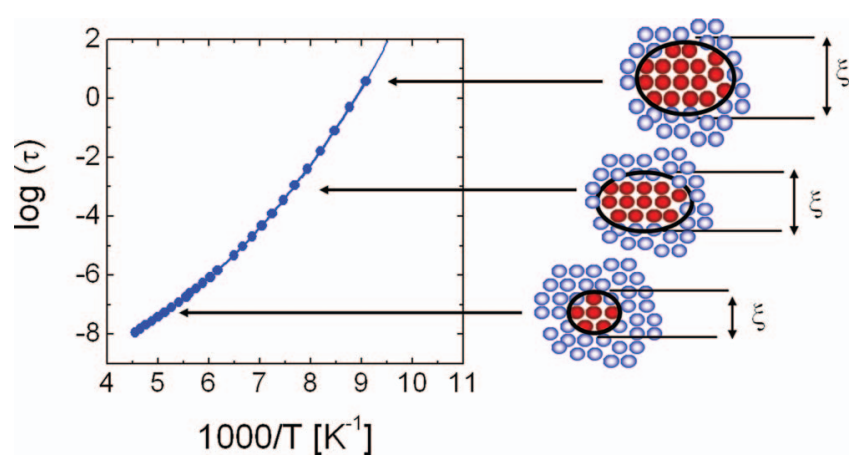

FIG. 5. Generic relaxation map showing the temperature dependence of the $\alpha$-relaxation. The temperature at which this process reaches a relaxation time of $100 \mathrm{~s}(\log (\tau)=2)$ is defined as the glass transition temperature $\left(T_{g}\right)$. In the figure a schematic illustration of the hypothetical cooperatively rearranged region (CRR), and how its size increases with decreasing temperature, is given.

ity of the dynamic glass transition. ${ }^{43}$ Different models have been proposed to explain the non-Arrhenius behavior of the $\alpha$-relaxation of glass forming liquids that all assume that the liquid consists of regions which are dynamically correlated. According to Adam and Gibbs, ${ }^{45}$ glass-formers are characterized by a self-organization of the molecules in the liquid, forming cooperatively rearranged regions (CRR). A CRR is defined as a subsystem, which can rearrange into another configuration, independently of its environment. The CRR size is small at high temperatures and increases with decreasing temperatures. Approaching the glass transition the size of the CRR becomes infinitely large (see cartoon in Figure 5) and a temperature dependence described by the VFT law can be deduces.

The CRR size can be described by a characteristic length, $\xi$, that increases with decreasing temperature until the cooperative region comprises the whole system causing the freezing of mobility at $T_{o}$ (see cartoon in Figure 5). The characteristic length of the CRR at $T_{g}$ was estimated to be $1-4 \mathrm{~nm}$, by different experimental techniques, ${ }^{46-51}$ for several materials (polymers and glass forming systems). In this picture, confinement induces a crossover in the temperature dependence of the relaxation time when the CRR size reaches the characteristic confinement length. Below this temperature the CRR would remain constant and Arrhenius-like temperature dependence is expected.

Taking the above arguments into account, process 2 could be assigned to the $\alpha$-relaxation of water confined in $\mathrm{C}-\mathrm{S}-\mathrm{H}$ gel. Then, the crossover observed can be understood as an onset of finite size effects. At high temperatures ( $\mathrm{T}>145 \mathrm{~K}$ ) the CRR size is smaller than the size of the confinement and a VFT-like temperature dependence of the relaxation time is observed. When the temperature is decreased (and the size of the CRR is expected to increase) the presence of confinement walls results in a limitation of the CRR growth. Consequently, an Arrhenius behavior of the relaxation time is detected at lower temperatures. Since the calorimetric glass transition temperature $\left(T_{g}\right)$ corresponds to the VFT extrapolation to relaxation time in the order of $100 \mathrm{~s}$, it would not be experimentally detectable by DSC. A similar behavior has 
already been observed for several polymers confined under nanometric conditions. ${ }^{43,52-54}$

The presence of such a crossover (associated with confinement effects) for water dynamics in well-defined confinement systems has been observed in different types of systems. Water molecules confined in graphite oxide ${ }^{55}$ show a crossover at about $180 \mathrm{~K}$, water in molecular sieves at $185 \mathrm{~K}^{56}$ and water confined in MCM-41 at $180 \mathrm{~K} .{ }^{10}$ Nevertheless, it is important to note that when water molecules do not suffer from confinement effect, the crossover is not generally observed. Instead, the temperature dependence of the relaxation time is shown to be of Arrhenius type in the whole temperature range. The absence of such a confinement-related crossover was recently reported in the case of water around silica particles $\left(\mathrm{SiO}_{2}\right),{ }^{57}$ and on rutile $\left(\mathrm{TiO}_{2}\right.$ particles $){ }^{58} \mathrm{In}-$ terestingly, an Arrhenius behavior over the full range of experimental temperatures was also observed for a monolayer of water confined in MCM41. ${ }^{59}$ Note that the main difference between C-S-H gel and silica particles is the fact that in the gel, water is confined in nano-pores of different sizes, whereas on the surface of the particles $\left(\mathrm{SiO}_{2}\right.$ or $\left.\mathrm{TiO}_{2}\right)$ this restriction does not emerge since water molecules are located on the external surface.

\section{Relaxation map - Comparison between BDS- and QENS-experiments}

As obvious from Figure 2(b), the temperature dependence of the relaxation time that is obtained by fitting the dielectric data only in a restricted frequency interval $\left(10^{6}-10^{8} \mathrm{~Hz}\right)$ resembles that obtained by QENS. ${ }^{6}$ We thus get a relaxation map where this process changes from a low temperature Arrhenius behavior to non-Arrhenius dependence at higher temperatures. The change in temperature dependence is observed as an abrupt crossover at about $220 \mathrm{~K}$. The crossover together with an only smoothly variation of the CC (Eq. (1)) fitting parameters (relaxation strength $(\Delta \varepsilon)$ and symmetric shape parameter $(\alpha)$ in the entire temperature range (Figure 2(c)) gives the impression of one single relaxation process that changes its temperature dependence around $220 \mathrm{~K}$. As a consequence, by taking only this limited frequency interval in consideration, it is possible to believe in the existence of a fragile-to-strong transition displayed by an abrupt crossover in the temperature dependence of the relaxation process. However, by taking a broader frequency interval into account, a different scenario is obtained (Figure 3). In contrast to the sudden change in temperature dependence as obtained by QENS (and also by BDS in the limited frequency interval) the relaxation process is well described by an Arrhenius temperature dependence in the entire temperature range where it is visible. However, as seen in Figure 3(b), in the temperature interval where the apparent crossover occurs, two different relaxation processes (1 and 2) are approaching in time. At low temperatures $(\mathrm{T}<180 \mathrm{~K})$ these two processes are well separated in frequency, whereas at higher temperatures they become very close to each other. So, if the analysis is performed in a restricted frequency interval, only the faster relaxation is observed and, consequently, the crossover is produced. Thus, the crossover is only a merging between two different processes. This behavior is also in agreement with a recent study on $\mathrm{LiCl}$ solution by dielectric spectroscopy and dynamic light scattering ${ }^{20}$ where it was shown that the fragileto-strong transition is only an effect of the limited resolution obtained by QENS. The effect of resolution and the choice of analysis method were also discussed by Doster et al. ${ }^{17}$ in a recent QENS study on protein hydration water. Thus, for water confined in various systems, including C-S-H gel, the apparent crossover is not due to a fragile-to-strong transition. Rather it is a consequence of that the analysis is performed in a limited frequency interval, which at least for the C-S-H gel is the merging region of two different relaxation processes, which occur at temperatures above $210 \mathrm{~K}$.

\section{Possible molecular origin of the fastest relaxation in C-S-H}

As mentioned above, the origin of the fastest relaxation process (process 1) observed in C-S-H gel is not obvious. At very low temperatures $(130-150 \mathrm{~K})$ it appears as an excess wing, and it can thus not be resolved as a separate peak, whereas at higher temperatures (above $200 \mathrm{~K}$ ) it emerges as a well-resolved peak. In our previous study we observed ${ }^{35}$ that the relaxation strength of this fastest process is very small compared to that of the main peaks (processes 2 and 3) and that its intensity slightly depends on the water content. Therefore, we have, stepwise, removed most of the water from the $\mathrm{C}-\mathrm{S}-\mathrm{H}$ gel by drying it under an inert atmosphere (inside the dielectric cryostat) at different temperatures $(120,200,250$, and $350{ }^{\circ} \mathrm{C}$ ) during one hour in order to study the origin of this fast relaxation process. The inert atmosphere is important since it ensures that neither carbonatation nor other reactions occur in the gel. ${ }^{60}$ After each drying cycle, a loss spectrum was collected. In Figure 6 the result is shown for $\mathrm{T}=130 \mathrm{~K}$. From this figure, it is observed that processes 2 and 3 disappear even at the lowest drying temperature $\left(120^{\circ} \mathrm{C}\right)$. This is an expected behavior since it is generally considered that free water molecules evaporate when heating/drying cementitious materials at $105^{\circ} \mathrm{C} .{ }^{61}$ On the

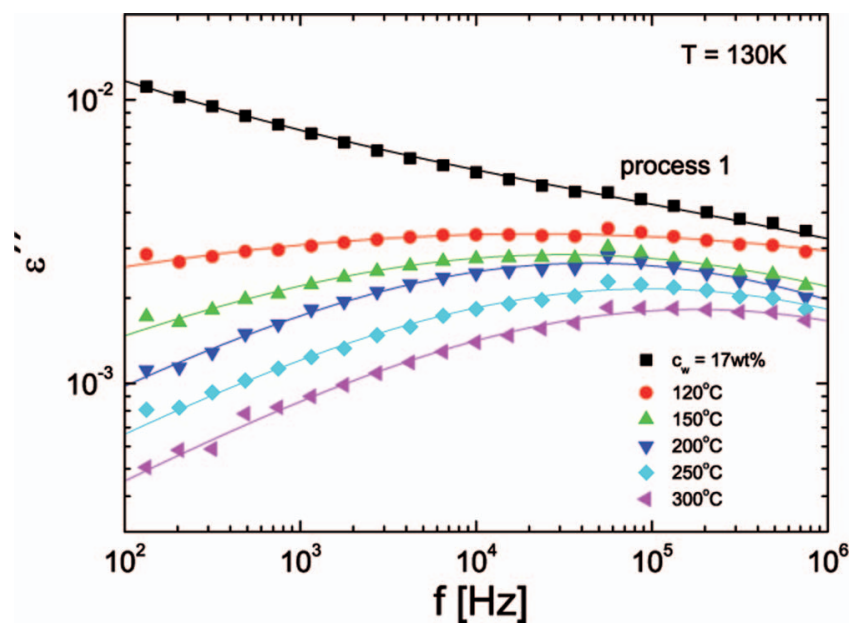

FIG. 6. Loss component $\left(\varepsilon^{\prime \prime}\right)$ of the complex dielectric permittivity at $130 \mathrm{~K}$, after drying at different temperatures $\left(120,200,250\right.$, and $\left.350{ }^{\circ} \mathrm{C}\right)$ during one hour. 
contrary, the fastest process (process 1) is still present after drying at very high temperatures $\left(350^{\circ} \mathrm{C}\right)$. Thus, even if the intensity of process 1 varies with water content (see Figure B in the supplementary material ${ }^{42}$ ) it is not likely that its origin is due to motion of water molecules since this fastest relaxation remains also after extensive drying even at high temperatures.

From Figure 6 it is, furthermore, obvious that the intensity of process 1 is somewhat decreased with increased drying temperature and that the relaxation becomes slightly faster. The calculated activation energy (Eq. (2)) associated with this process is $0.24 \pm 0.03 \mathrm{eV}$, for all the drying temperatures analyzed. The fact that this value is somewhat higher than the corresponding value obtained for the water containing sample (CSH-22) indicates that the water molecules are acting as a plasticizer in the non-dried samples. Thus, even if the origin of process 1 cannot be attributed to a pure water relaxation it seems that the water acts as an amplification of the signal. Therefore, we have to look for other polar groups in our sample, or preferably polar groups at the surfaces of the C-S-H gel to which water can bind (or be released with increasing temperature). The surface of the C-S-H gel contains both silanol $(\mathrm{Si}-\mathrm{OH})$ and calcium hydroxide $(\mathrm{Ca}-\mathrm{OH})$ groups. ${ }^{60,62-64}$ These groups are both polar and have an active dipole moment $(1.5 \mathrm{D})$ comparable to that of the water molecule $(1.85 \mathrm{D})$. As dielectric spectroscopy is probing the fluctuations of dipole moments, our results could thus be an indication on that the fastest process is due to the reorientation of these hydroxyl groups $(\mathrm{OH})$. This view is also supported by the value of the activation energy $(0.24 \mathrm{eV})$ founded for this process, which is very close to the energy required to break a single hydrogen bond $(0.22 \mathrm{eV}){ }^{65}$ Such an interpretation is furthermore consistent with an earlier study on high temperature treated silica gel, where a fast process with similar activation energy was attributed to the rotation of surface bonded hydroxyl groups. ${ }^{66} \mathrm{~A}$ similar result was also obtained for water in montmorillonite (hydrated clays) ${ }^{67}$ and more recently in hydrated ordinary Portland cement where a decrease in the elastic intensity occurs at about $190 \mathrm{~K}$ and attributed to the activation of $\mathrm{OH}^{-}$motions. ${ }^{68}$ In addition, studies on dried amorphous polysaccharides (cellulose and dextran) with two and three hydroxyl groups per ring, respectively, have shown on a weak relaxation similar to the here obtained process 1 , however, with a somewhat higher activation energy of $0.33 \mathrm{eV}^{69}$ Intriguingly, a similar fast process with a relatively low value of the activation energy is also found in other systems containing large amounts of hydroxyl groups such as, for instance, MCM-41 and various biological systems. ${ }^{11,70,71}$

However, even if it cannot be established from the present study that process 1 in C-S-H gel has the same molecular origin as water in various types of hydrophilic confining systems, it is interesting to compare the results with the behavior of water in other types of hydrophilic systems containing large amounts of hydroxyl groups. For instance, in a previous study on $\mathrm{D}_{2} \mathrm{O}$ in MCM- $41^{72}$ it was found that water exhibits a crossover from a high temperature VFT to a low temperature Arrhenius behavior, where the low temperature process has about the same activation energy as the here observed process 1. This scenario is thus in agreement with what was found for water in C-S-H gel by analyzing only a limited frequency interval (see Figure 2(b)). On the other hand, in a study on water in MCM-41 by dielectric spectroscopy there was no sign of a transition. ${ }^{73}$ In that study the fastest dielectric process was shown to follow an Arrhenius temperature dependence in the entire temperature interval where it was observable. Thus, a similar behavior as was found when analyzing the water containing C-S-H gel in a broader frequency interval (see Figure 3(b)). Moreover, from a study on $\mathrm{D}_{2} \mathrm{O}$ in MCM- $41^{74}$ it was suggested that the crossover occurs in the central region of the pores and that the water close to the pore walls does not participate in the transition. This scenario would then be in accordance with our interpretations on surface bonded hydroxyl groups but, however, in contrast to the results obtained for a copper-based hydrate ${ }^{75}$ that showed that it is the relaxation time of water condensed on the surfaces that deviate at low temperatures. Thus, even if it is perfectly clear that process 1 is not due to the relaxation of water itself, we can only speculate about the origin of this process from the present results. More detailed studies are needed to elucidate the origin of the fastest process in C-S-H gel and its possible relation to the fast weak relaxation found in various types of hydrophilic water containing systems.

\section{CONCLUSION}

The present dielectric study on the dynamics of water in $\mathrm{C}-\mathrm{S}-\mathrm{H}$ gel shows no sign of the fragile-to-strong transition as previously has been indicated by QENS for a similar system. Instead the apparent crossover in the temperature dependence is here shown to be due to that the analysis is performed in a very limited frequency interval, which, in turn, masks the merging between the main water relaxation and a faster relaxation process. This is also consistent with recent literature data ${ }^{16-19}$ on other systems.

When the pore water in C-S-H gel is dried out, it is shown that processes 2 and 3 (both originate from the dynamics of water molecules) disappear whereas the fastest process 1 remains. Due to this, process 1 could be attributed to the nonremovable and dielectrically active hydroxyl groups on the surface of the C-S-H gel.

\section{ACKNOWLEDGMENTS}

The support from the Basque Government through the Nanoiker project (Grant No. E11-304) under the ETORTEK Program is acknowledged. S.C. also acknowledges the support of the Spanish Ministry of Education, project MAT201231088 and the Basque Government, and project IT-654-13 and Professor J. Colmenero for helpful discussions. H.J. acknowledges the Swedish Research Council Formas for financial support.

${ }^{1}$ P. Ball, Chem. Rev. 108, 74 (2008); P. G. Debenedetti and H. E. Stanley, Phys. Today 56(6), 40 (2003).

${ }^{2}$ P. H. Poole, F. Sciortino, U. Essmann, and H. E. Stanley, Nature (London) 360, 324 (1992).

${ }^{3}$ O. Mishima and H. E. Stanley, Nature (London) 396, 329 (1998). 
${ }^{4}$ L. Liu, S. H. Chen, A. Faraone, C. W. Yen, and C. Y. Mou, Phys. Rev. Lett. 95, 117802 (2005).

${ }^{5}$ E. Mamontov, C. J. Burnham, S. H. Chen, A. P. Moravsky, C. K. Loong, N. R. de Souza, and A. I. Kolesnikov, J. Chem. Phys. 124, 194703 (2006).

${ }^{6}$ Y. Zhang, M. Lagi, F. Ridi, E. Fratini, P. Baglioni, E. Mamontov, and S. H. Chen, J. Phys.: Condens. Matter 20, 502101 (2008).

${ }^{7}$ S. H. Chen, L. Liu, X. Chu, Y. Zhang, E. Fratini, P. Baglioni, A. Faraone, and E. Mamontov, J. Chem. Phys. 125, 171103 (2006).

${ }^{8}$ X. Chu, E. Fratini, P. Baglioni, A. Faraone, and S. H. Chen, Phys. Rev. E 77, 011908 (2008).

${ }^{9}$ S. H. Chen, L. Liu, E. Fratini, P. Baglioni, A. Faraone, and E. Mamontov, Proc. Natl. Acad. Sci. U.S.A. 103, 9012 (2006).

${ }^{10}$ J. Sjöström, J. Swenson, R. Bergman, and S. Kittaka, J. Chem. Phys. 128, 154503 (2008).

${ }^{11}$ J. Swenson, H. Jansson, and R. Bergman, Phys. Rev. Lett. 96, 247802 (2006); H. Jansson and J. Swenson, Biochim. Biophys. Acta 1804, 20 (2010).

${ }^{12}$ S. Capaccioli, K. L. Ngai, S. Ancherbak, P. A. Rolla, and N. Shinyashiki, J. Non-Cryst. Solids 357, 641 (2011).

${ }^{13}$ K. Grzybowska, M. Paluch, A. Grzybowski, S. Pawlus, S. Ancherbak, D. Prevosto, and S. Capaccioli, J. Phys. Chem. Lett. 1, 1170 (2010).

${ }^{14}$ S. Cerveny, J. Colmenero, and A. Alegria, J. Non-Cryst. Solids 353, 4523 (2007); S. Cerveny, J. Colmenero, and A. Alegria, Eur. Phys. J. Spec. Top. 141, 49 (2007).

${ }^{15}$ S. Cerveny, J. Colmenero, and A. Alegria, Phys. Rev. E 77, 031803 (2008).

${ }^{16}$ M. Vogel, Phys. Rev. Lett. 101, 225701 (2008).

${ }^{17}$ W. Doster, S. Busch, A. M. Gaspar, M. S. Appavou, J. Wuttke, and H. Scheer, Phys. Rev. Lett. 104, 098101 (2010).

${ }^{18}$ S. Pawlus, S. Khodadadi, and A. P. Sokolov, Phys. Rev. Lett. 100, 108103 (2008).

${ }^{19}$ S. Khodadadi, S. Pawlus, and A. P. Sokolov, J. Phys. Chem. B 112, 14273 (2008).

${ }^{20}$ N. Nakanishi, P. Griffin, E. Mamontov, and A. P. Sokolov, J. Chem. Phys. 136, 124512 (2012).

${ }^{21}$ S. Capponi, A. Arbe, S. Cerveny, R. Busselez, B. Frick, J. P. Embs, and J. Colmenero, J. Chem. Phys. 134, 204906 (2011).

${ }^{22}$ R. Busselez, A. Arbe, S. Cerveny, S. Capponi, and J. Colmenero, J. Chem. Phys. 137, 084902 (2012).

${ }^{23}$ J. M. Bullard, H. M. Jennings, R. A. Livingston, G. W. Scherer, J. S. Schweitzer, K. L. Scrivener, and K. L. J. J. Thomas, Cem. Concr. Res. 41, 1208 (2011)

${ }^{24}$ J. J. Thomas, J. J. Biernacki, J. W. Bullard, S. Bishnoi, J. S. Dolado, G. W. Scherer, and A. Luttge, Cem. Concr. Res. 41, 1257 (2011).

${ }^{25}$ L. P. Aldridge, H. N. Bordallo, and A. Desmedt, Physica B - Cond. Matter 350, E565 (2004).

${ }^{26}$ H. Manzano, S. Moeini, F. Marinelli, A. C. T. van Duin, F. J. Ulm, and R. J. M. Pellenq, J. Am. Chem. Soc. 134, 2208 (2012).

${ }^{27}$ C. Powers and T. L. Brownyard, Proc. Am. Concrete Inst. 43, 971-992 (1947), Bulletin No. 22, Portland Cement Association (March 1948).

${ }^{28}$ R. F. Feldman and P. J. Sereda, Engl. J. Canada 53, 53 (1970).

${ }^{29}$ P. J. Sereda, R. F. Feldman, and V. S. Ramachandran, Proceeding of the Seventh International Congress on Chemistry of Cement VI-1/3-VI-1/4, 1980.

${ }^{30}$ H. M. Jennings, Cem. Concr. Res. 30, 101 (2000).

${ }^{31}$ H. M. Jennings, Cem. Concr. Res. 38, 275 (2008).

${ }^{32}$ I. G. Richardson and G. W. Groves, Cem. Concr. Res. 22, 1001 (1992).

${ }^{33}$ J. S. Dolado, M. Griebel, J. Hamaekers, and F. Heber, J. Mater. Chem. 21, 4445 (2011).

${ }^{34}$ J. S. Dolado and K. van Breugel, Cem. Concr. Res. 41, 711 (2011).

${ }^{35}$ S. Cerveny, S. Arrese-Igor, J. S. Dolado, J. J. Gaitero, A. Alegría, and J. Colmenero, J. Chem. Phys. 134, 034509 (2011).

${ }^{36}$ F. Ridi, P. Luciani, E. Fratini, and P. Baglioni, J. Phys. Chem. B 113, 3080 (2009)

${ }^{37}$ R. H. Cole and K. S. Cole, J. Chem. Phys. 10, 98 (1942).

${ }^{38}$ A. Arbe, A. Alegria, J. Colmenero, S. Hoffmann, L. Willner, and D. Richter, Macromolecules 32, 7572 (1999).

${ }^{39}$ K. Elamin, S. Cazzato, J. Sjostrom, S. M. King, and J. Swenson, J. Phys. Chem. B 117, 7363 (2013).
${ }^{40}$ H. Vogel, Phys. Z. 22, 645 (1921); G. S. Fulcher, J. Am. Chem. Soc. 8, 339 (1925); G. Tammann and W. Z. Hesse, Anorg. Allg. Chem. 156, 245 (1926).

${ }^{41}$ H. F. Taylor, Cement Chemistry (Academic, New York, 1990).

${ }^{42}$ See supplementary material at $\mathrm{http} / / / \mathrm{dx}$.doi.org/10.1063/1.4826638 for the temperature dependence of the relaxation times at different water concentrations.

${ }^{43}$ A. Schönhals, H. Goering, C. Schick, B. Frick, and R. Zorn, Eur. Phys. J. E 12, 173 (2003).

${ }^{44}$ A. R. Bras, E. G. Merino, P. D. Neves, I. M. Fonseca, M. Dionísio, A. Schönhals, and N. T. Correia, J. Phys. Chem. C 115, 4616 (2011).

${ }^{45}$ G. Adam and J. H. Gibbs, J. Chem. Phys. 43, 139 (1965).

${ }^{46}$ E. W. Fischer, E. Donth, and W. Steffen, Phys. Rev. Lett. 68, 2344 (1992).

${ }^{47}$ E. Donth, The Glass Transition: Relaxation Dynamics in Liquids and Disordered Materials (Springer, Berlin, Germany, 2001).

${ }^{48}$ L. Berthier, G. Biroli, J.-P. Bouchaud, L. Cipelletti, D. El Masri, D. L'Hote, F. Ladieu, and M. Pierno, Science 310, 1797 (2005).

${ }^{49}$ G. A. Schwartz, D. Cangialosi, A. Alegria, and J. Colmenero, J. Chem. Phys. 124, 154904 (2006).

${ }^{50}$ G. A. Schwartz, A. Alegria, and J. Colmenero, J. Chem. Phys. 127, 154907 (2007).

${ }^{51}$ D. Cangialosi, A. Alegría, and J. Colmenero, Phys. Rev. E 80, 041505 (2009).

${ }^{52}$ G. Floudas, S. Paraskeva, N. Hadjichristidis, G. Fytas, B. Chu, and A. N. Semenov, J. Chem. Phys. 107, 5502 (1997).

${ }^{53}$ G. Turky, D. Wolff, and A. Schönhals, Macromol. Chem. Phys. 213, 2420 (2012).

${ }^{54}$ C. Iacob, J. R. Sangoro, W. K. Kipnusu, R. Valiullin, J. Kärger, and F. Kremer, Soft Matter 8, 289 (2012).

${ }^{55}$ S. Cerveny, F. Barroso-Bujans, A. Alegria, and J. Colmenero, J. Phys. Chem. C 114, 2604 (2010).

${ }^{56}$ H. Jansson and J. Swenson, Eur. Phys. J. E 12, 51 (2003).

${ }^{57}$ S. Cerveny, G. A. Schwartz, J. Otegui, J. Colmenero, J. Loichen, and S. Westermann, J. Phys. Chem. C 116, 24340 (2012).

${ }^{58}$ E. Mamontov, L. Vlcek, D. J. Wesolowski, P. T. Cummings, J. Rosenqvist, W. Wang, D. R. Cole, L. M. Anovitz, and G. Gasparovic, Phys. Rev. E 79, 51504 (2009).

${ }^{59}$ C. E. Bertrand, K.-H. Liu, E. Mamontov, and S.-H. Chen, Phys. Rev. E 87, 042312 (2013)

${ }^{60}$ P. C. Fonseca and H. M. Jennings, Cem. Concr. Res. 40, 1673 (2010).

${ }^{61}$ H. N. Bordallo, L. P. Aldridge, P. Fouquet, L. C. Pardo, T. Unruh, J. Wuttke, and F. Yokaichiya, ACS App. Mater. Interfaces 1, 2154 (2009).

${ }^{62}$ A. Ayuela, J. S. Dolado, I. Campillo, Y. R. De Miguel, E. Erklzia, D. Sánchez-Portal, A. Rubio, and P. M. Echenique, J. Chem. Phys. 127, 164710 (2007).

${ }^{63}$ P. Rejmak, J. S. Dolado, M. J. Stott, and A. Ayuela, J. Phys. Chem. C 116, 9755 (2012).

${ }^{64}$ J. J. Thomas, J. Chen, H. M. Jennings, and D. A. Neumann, Chem. Mater. 15, 3813 (2003).

${ }^{65} \mathrm{~L}$. Pauling, The Natural of the Chemical Bond and the Structure of Molecules and Crystal (Cornell University Press, New York, 1960), Chap. 12.

${ }^{66}$ S. Kondo and M. Muroya, Bull. Chem. Soc. Jpn. 42, 1165 (1969).

${ }^{67}$ W. P. Gates, H. N. Bordallo, L. P. Aldridge, T. Seydel, H. Jacobsen, V. Marry, and G. J. Churchman, J. Phys. Chem. C 116, 5558 (2012).

${ }^{68}$ J. Jacobsen, M. S. Rodrigues, M. T. F. Telling, A. L. Beraldo, S. F. Santos, L. P. Aldridge, and H. N. Bordallo, Nat. Sci. Rep. 3, 2667 (2013).

${ }^{69} \mathrm{H}$. Monte and J. Y. Cavaille, Polymer 40, 2649 (1999).

${ }^{70}$ J. Swenson, H. Jansson, J. Hedström, and R. Bergman, J. Phys. Condens. Matter 19, 205109 (2007).

${ }^{71}$ P. Berntsen, C. Svanberg, and J. Swenson, J. Phys. Chem. B 115, 1825 (2011).

${ }^{72}$ K. Yoshida, T. Yamaguchi, S. Kittaka, M. C. Bellissent-Funel, and P. Fouquet, J. Chem. Phys. 129, 054702 (2008).

${ }^{73}$ J. Hedstrom, J. Swenson, R. Bergman, H. Jansson, and S. Kittaka, Eur. Phys. J. Spec. Top. 141, 53 (2007).

${ }^{74}$ K. Yoshida, T. Yamaguchi, S. Kittaka, M. C. Bellissent-Funel, and P. Fouquet, J. Phys.: Condens. Matter 24, 064101 (2012).

${ }^{75}$ T. Yamada, R. Yonamine, T. Yamada, H. Kitagawa, M. Tyagi, M. Nagao, and O. Yamamuro, J. Phys. Chem. B 115, 13563 (2011). 